\title{
Síndrome moyamoya y enfermedad de Graves en una mujer joven
}

\section{Moyamoya syndrome and Graves' disease in a young woman}

\author{
Jairo Lizarazo, Freddy Niño, Hugo Alvarado, Noé Castro• \\ CÚCUTA (COLOMBIA)
}

\section{Resumen}

Introducción: la enfermedad y el síndrome moyamoya son entidades cerebrovasculares caracterizadas por la estenosis u oclusión de la porción terminal de la arteria carótida interna y la porción proximal de las arterias cerebrales media y anterior, acompañada por la formación de vasos colaterales en forma de red. La enfermedad de Graves es una tiroiditis autoinmune de origen desconocido y es la causa más común de hipertiroidismo. La asociación de estas dos enfermedades es rara.

Caso clínico: mujer de 27 años de edad hospitalizada por un infarto cerebral e hipertiroidismo debido a enfermedad de Graves. Las imágenes diagnósticas identificaron un infarto cerebral extenso temporoparietal derecho en territorio de la arteria cerebral media. Además, se evidenció la estenosis de la porción supraclinoidea de la arteria carótida interna derecha y de la porción proximal de las arterias cerebral media y anterior derechas, sin presencia de vasos colaterales. Inicialmente, fue tratada con antitiroideos y esteroides. Requirió dos tratamientos con yodo radiactivo para controlar el hipertiroidismo. La lesión cerebral le dejó como secuelas déficit motor y sensitivo izquierdos y una epilepsia focal sintomática controlada con medicación. Actualmente recibe terapia de suplencia tiroidea y anticonvulsivante. El seguimiento angiográfico cerebral demostró aumento de la estenosis de los vasos cerebrales afectados sin evidencia de nuevas lesiones cerebrales

Conclusiones: los pacientes con hipertiroidismo secundario a la enfermedad de Graves y manifestaciones clínicas neurológicas focales agudas deben investigarse para un posible síndrome moyamoya. (Acta Med Colomb 2013; 38: xx-xx).

Palabras clave: síndrome moyamoya, enfermedad moyamoya, hipertiroidismo, tirotoxicosis, enfermedad de Graves, infarto cerebral.

\section{Abstract}

Introduction: the disease and moyamoya syndrome are cerebrovascular entities characterized by stenosis or occlusion of the terminal portion of the internal carotid artery and the proximal portion of the middle and anterior cerebral arteries, accompanied by the formation of collateral vessels in the form of network. Graves' disease is an autoimmune thyroiditis of unknown origin and is the most common cause of hyperthyroidism. The association of these two diseases is rare.

Case report: 27 year-old woman hospitalized for a stroke and hyperthyroidism due to Graves' disease. Diagnostic imaging identified a large right temporoparietal infarct in the territory of the middle cerebral artery. Additionally, stenosis of the supraclinoid portion of the right internal carotid artery and the proximal portion of the anterior and middle right cerebral arteries without the presence of collateral vessels, was evidenced. Initially, she was treated with antithyroid drugs and steroids. Two treatments with radioactive iodine to control hyperthyroidism were needed. The brain injury left sequelae of left motor and sensory deficits and symptomatic focal epilepsy, controlled by medication. She is currently receiving thyroid substitution therapy and anticonvulsant therapy. Angiographic follow-up showed increased vessel stenosis of the cerebral affected vessels without evidence of new brain lesions.

Conclusions: patients with hyperthyroidism secondary to Graves' disease and acute focal neurological manifestations should be investigated for possible moyamoya syndrome. (Acta Med Colomb 2013; 38: xx-xx).

Keywords: moyamoya syndrome, moyamoya disease, hyperthyroidism, thyrotoxicosis, Graves disease, stroke.
Dres. Jairo Lizarazo, Freddy Niño, Hugo Alvarado, Noé Castro: Departamento de Medicina Interna, Hospital Universitario Erasmo Meoz de Cúcuta, Colombia Correspondencia. Dr. Jairo Lizarazo, Cúcuta, Norte de Santander (Colombia). E-mail: jfilizar@gmail.com Recibido: 27/11/2012 Aceptado: 18/09/2013 


\section{Introducción}

La enfermedad moyamoya es una entidad caracterizada por la estenosis u oclusión de la porción terminal de la arteria carótida interna y la porción proximal de las arterias cerebrales media y anterior, acompañada por la formación de vasos colaterales en forma de red. La causa de esta enfermedad es desconocida y se deben descartar otras etiologías para establecer el diagnóstico definitivo. El término síndrome moyamoya, se usa para aquellos casos que tienen hallazgos angiográficos similares a los de la enfermedad moyamoya y una enfermedad sistémica asociada (1). Los pacientes con hallazgos vasculares unilaterales tienen el síndrome moyamoya, aun si no tienen factor de riesgo asociado (2).

La enfermedad de Graves es un trastorno autoinmune, en el cual autoanticuerpos contra el receptor de la hormona estimulante del tiroides (TSH) inducen la estimulación continua de la glándula tiroides sin retroalimentación negativa, lo que genera hipertiroidismo. La coexistencia de síndrome moyamoya y enfermedad de Graves es rara (1).

Presentamos el caso de una mujer joven con enfermedad de Graves a quien se le diagnosticó el síndrome moyamoya luego de sufrir un infarto cerebral.

\section{Caso clínico}

Se trata de una mujer de 27 años de edad, natural de Hacarí (Norte de Santander) y procedente de Ocaña (Norte de Santander) que ingresó por urgencias al Hospital Universitario Erasmo Meoz (HUEM) el 3/5/2008, remitida por cuadro clínico de dos días de evolución, caracterizado por pérdida de la fuerza del hemicuerpo izquierdo. Además, presentaba desde hacía varios días exoftalmos, palpitaciones y nerviosismo.

En junio de 2007 presentó bocio y a partir de septiembre de 2007, ansiedad, taquicardia y exoftalmos bilateral. El 4/10/2007 un médico internista le hace el diagnóstico de hipertiroidismo segundario a la enfermedad de Graves, y le formuló metimazol $20 \mathrm{mg}$ vo cada ocho horas y metoprolol $50 \mathrm{mg}$ vo cada 12 horas. En febrero de 2008 fue valorada por endocrinólogo del HUEM encontrándole un bocio nodular difuso de unos $100 \mathrm{~g}$ de peso, le confirma el diagnóstico de enfermedad de Graves y el tratamiento iniciado y la cita en dos meses para la administración de I131. La paciente no cumplió la cita, ni recibió la terapia antitiroidea en forma regular. No tenía otros antecedentes de importancia. Su historia obstétrica era G0P0A0.

En el examen físico de ingreso se encontró una paciente con los siguientes signos vitales: tensión arterial 140/90 $\mathrm{mmHg}$, frecuencia cardiaca $92 \mathrm{lpm}$, frecuencia respiratoria 16 por minuto y temperatura de $36.7^{\circ} \mathrm{C}$. Se encontraba alerta, orientada, ansiosa, con lenguaje fluente, con lateralidad diestra. Se palpó un bocio tiroideo grado II y se observó un marcado exoftalmos bilateral sin diplopía, ni oftalmoparesia. Las pupilas eran de $3 \mathrm{~mm}$ con respuesta normal a la luz y a la acomodación. Tenía una paresia facial central izquierda, plejía flácida del miembro superior izquierdo y paresia (4/5) flácida del miembro inferior izquierdo, con reflejos miotáticos disminuidos y signo de Babinski positivo, además de hemihipoestesia del mismo lado. La marcha era parética y controlaba los esfínteres.

Los exámenes de laboratorio están consignados en la Tabla 1. La tomografía axial computadorizada (TAC) de cráneo simple (no mostrada) evidenciaba una lesión hipodensa temporoparietal derecha compatible con un infarto cerebral en territorio de la arteria cerebral media. Durante la hospitalización la paciente presentó cifras tensionales de hasta $170 / 100 \mathrm{mmHg}$ y episodios de agitación psicomotora. Además del tratamiento con metimazol y metoprolol, la paciente recibió yoduro de potasio cinco gotas VO cada 12 horas por siete días, sedación (inicialmente con olanzapina, luego con alprazolam), esteroides (inicialmente metilprednisolona $1 \mathrm{~g}$ IV diarios por tres días por sospecha inicial de vasculitis, luego prednisona $25 \mathrm{mg}$ vo diarios), aspirina, lovastatina y enoxaparina.

Tabla 1. Exámenes de laboratorio realizados durante la hospitalización inicial

\begin{tabular}{|c|c|}
\hline \multicolumn{2}{|l|}{ Cuadro hemático } \\
\hline Hemoglobina & $12,8 \mathrm{~g} / \mathrm{dL}$ \\
\hline Leucocitos & $9.400 / \mathrm{uL}$ \\
\hline PMN & $53,2 \%$ \\
\hline Linfocitos & $34,4 \%$ \\
\hline Monocitos & $11,9 \%$ \\
\hline Plaquetas & $204.000 / \mathrm{uL}$ \\
\hline TP INR & 1,66 \\
\hline \multicolumn{2}{|l|}{ Química sanguínea } \\
\hline Glicemia & $109 \mathrm{mg} / \mathrm{dL}$ \\
\hline Nitrógeno ureico & $15 \mathrm{mg} / \mathrm{dL}$ \\
\hline Creatinina sérica & $0,53 \mathrm{mg} / \mathrm{dL}$ \\
\hline \multicolumn{2}{|l|}{ Parcial de orina } \\
\hline Densidad & 1,020 \\
\hline $\mathrm{pH}$ & 6,0 \\
\hline Células bajas & 1-2 x campo \\
\hline Leucocitos & $15-20 \times$ campo \\
\hline Eritrocitos & 4-6 x campo \\
\hline Bacterias & + \\
\hline \multicolumn{2}{|l|}{ Hormonas tiroideas } \\
\hline TSH (rango normal: $0,4-4,0 \mu \mathrm{UI} / \mathrm{mL}$ ) & $0,05 \mu \mathrm{UI} / \mathrm{mL}$ \\
\hline T4 total (rango normal: $4,50-12,0$ ): & $21,24 \mu \mathrm{g} / \mathrm{dL}$ \\
\hline T4 libre (rango normal: $0,71-1,85$ ) & $4,09 \mathrm{ng} / \mathrm{dL}$ \\
\hline \multicolumn{2}{|l|}{ Estudios inmunológicos } \\
\hline Anticuerpos antinucleares & $1 / 320$ patrón moteado \\
\hline Anticuerpos anti ADN & Negativos \\
\hline Anticuerpos anti SM & Negativos \\
\hline Anticoagulante lúpico & Negativo \\
\hline Anticuerpos IgG anticardiolipina & Negativos \\
\hline Anticuerpos IgM anticardiolipina & Negativos \\
\hline \multicolumn{2}{|l|}{ Otros exámenes paraclínicos } \\
\hline Prueba de embarazo & Negativa \\
\hline Elisa para VIH & No reactiva \\
\hline VDRL & No reactiva \\
\hline EKG & Taquicardia sinusal. \\
\hline \multicolumn{2}{|l|}{ Eje QRS: $+60^{\circ}$} \\
\hline Radiografía del tórax & Normal \\
\hline Ecografía pélvica & Normal \\
\hline
\end{tabular}


Por sospecha de síndrome moyamoya se le solicitó angioresonancia cerebral (Figura 1a), realizada el 9/5/2008, la cual mostró estenosis distal de la arteria carótida interna derecha y proximal de las arterias cerebral anterior y media derechas. No se evidenciaron vasos colaterales. La condición clínica de la paciente se estabilizó y se le dio salida con metimazol, prednisona, propranolol y levomepromazina. Dos meses después reingresa al HUEM y el 12/7/2008 se le administró 25 mci de I131 por vía oral.

El 24/2/2009 se le hizo angiografía cerebral digital (figuras $2 \mathrm{a}$ y $2 \mathrm{~b}$ ) en donde se confirman los hallazgos del síndrome moyamoya unilateral derecho; las demás arterias cerebrales no presentaban lesiones. En junio y agosto de 2009 presentó sendas crisis epilépticas tonicoclónicas generalizadas con pérdida de la conciencia. Se le inició tratamiento con ácido valproico y se llevó hasta una dosis de $500 \mathrm{mg}$ VO cada ocho horas. El déficit motor izquierdo evolucionó hacia la hemiparesia espástica, con muy pobre recuperación de la fuerza del miembro superior y con una marcha helicópoda. Además, desarrolló alodinia en el miembro superior izquierdo y hombro izquierdo doloroso. Para sus secuelas neurológicas ha recibido tratamiento farmacológico e integral de rehabilitación con pobres resultados. También, ha requerido tratamiento psiquiátrico debido a una depresión reactiva.

En marzo de 2010 se le encontró hipertensión arterial y se le inició losartán $50 \mathrm{mg}$ vo cada 12 horas. Por recidiva del hipertiroidismo, se le indicó una segunda dosis

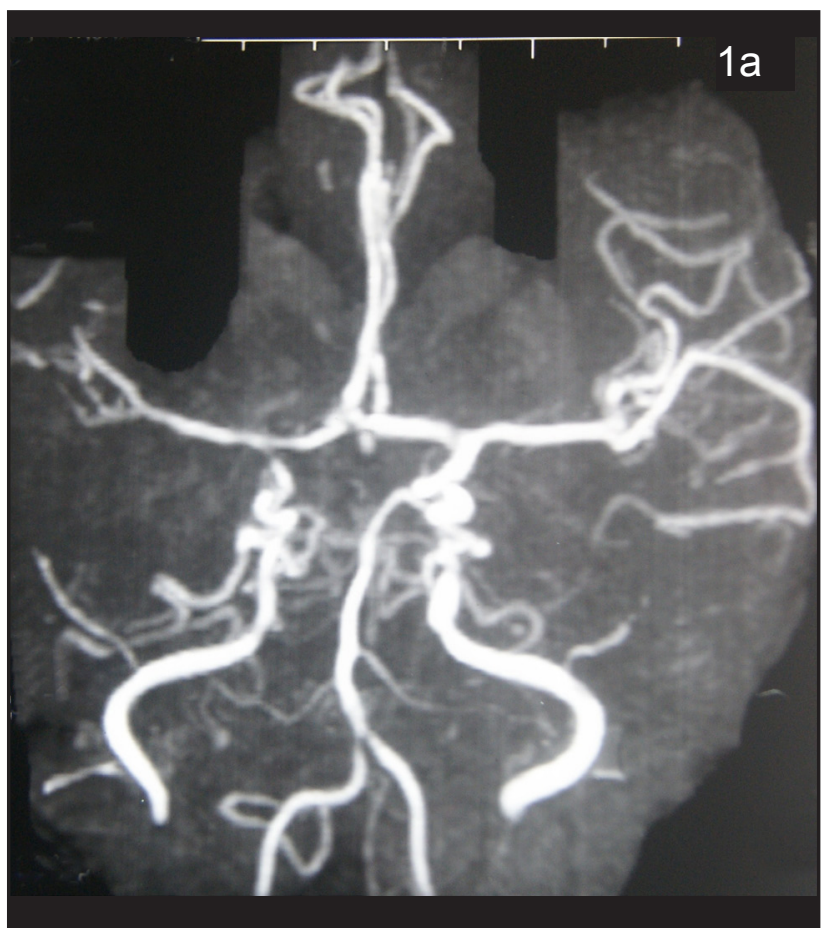

de yodoterapia la cual se hizo efectiva el 27/4/2010 con 50 mci de I131. En agosto de 2010 se confirmó el hipotiroidismo postratamiento con yodo radiactivo. Se le inició suplencia tiroidea con levotiroxina. La TAC de cráneo de control (18/10/2010, figura 3a) mostró encefalomalacia temporoparietal derecha y la segunda angiorresonancia cerebral (16/4/2011, Figura 1b) detectó una oclusión casi total de la porción proximal de la arteria cerebral anterior derecha cuyo territorio distal llena por la arteria cerebral anterior izquierda a través de la arteria comunicante anterior; existe aumento de la estenosis de la porción proximal de la arteria cerebral media derecha. No se evidenciaron vasos colaterales. Las imágenes por resonancia magnética del cerebro (16/4/2011, Figura 3b) detectaron la misma encefalomalacia temporoparietal derecha con dilatación compensatoria del ventrículo lateral del mismo lado.

En el último control (26/3/2012) la paciente se encontraba recibiendo terapia de suplencia tiroidea (levotiroxina $150 \mu \mathrm{g}$ vo diarios), ácido valproico $500 \mathrm{mg}$ vo cada ocho horas y el tratamiento sintomático del dolor. Las secuelas neurológicas permanecen estables y el exoftalmos mejoró totalmente.

\section{Discusión}

En general, la enfermedad y el síndrome moyamoya son poco frecuentes en el hemisferio occidental. En una encuesta realizada de la literatura en inglés entre los años 1972 y 1989 se encontraron 176 casos informados en América,

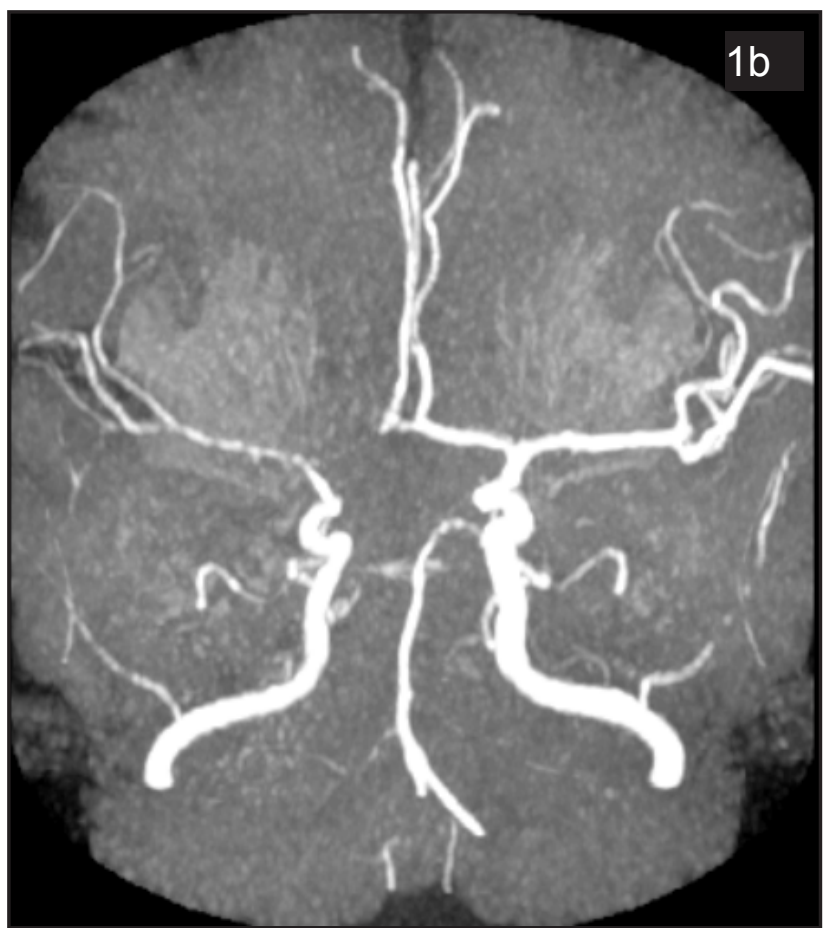

Figura 1. (a) Angiorresonancia cerebral (9/5/2008): estenosis de la porción supraclinoidea de la arteria carótida interna derecha y de la porción proximal de las arterias cerebral media y anterior derechas. (b) Angiorresonancia cerebral (16/4/2011): obliteración casi total de la porción proximal de la arteria cerebral anterior derecha, cuya porción distal llena a partir de la arteria cerebral anterior izquierda por la arteria comunicante anterior. Aumento de la estenosis de la porción proximal de la arteria cerebral media derecha. 

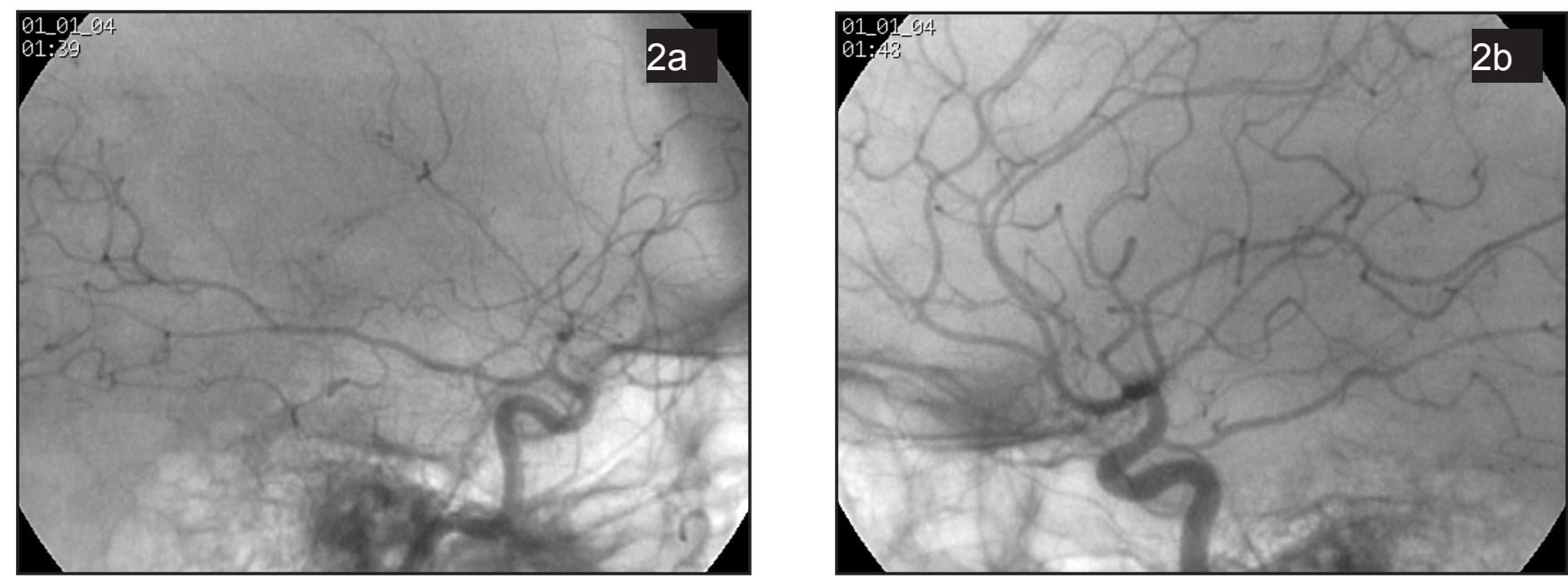

Figura 2. Angiografía cerebral digital (24/2/2009): (a) Arteriografía carotídea derecha muestra estenosis de la porción distal de la arteria carótida interna y de la porción proximal de las arterias cerebral anterior y media; hay disminución del número y el calibre de las ramas de las arterias cerebrales anterior y media. (b) Arteriografía carotídea izquierda: normal.
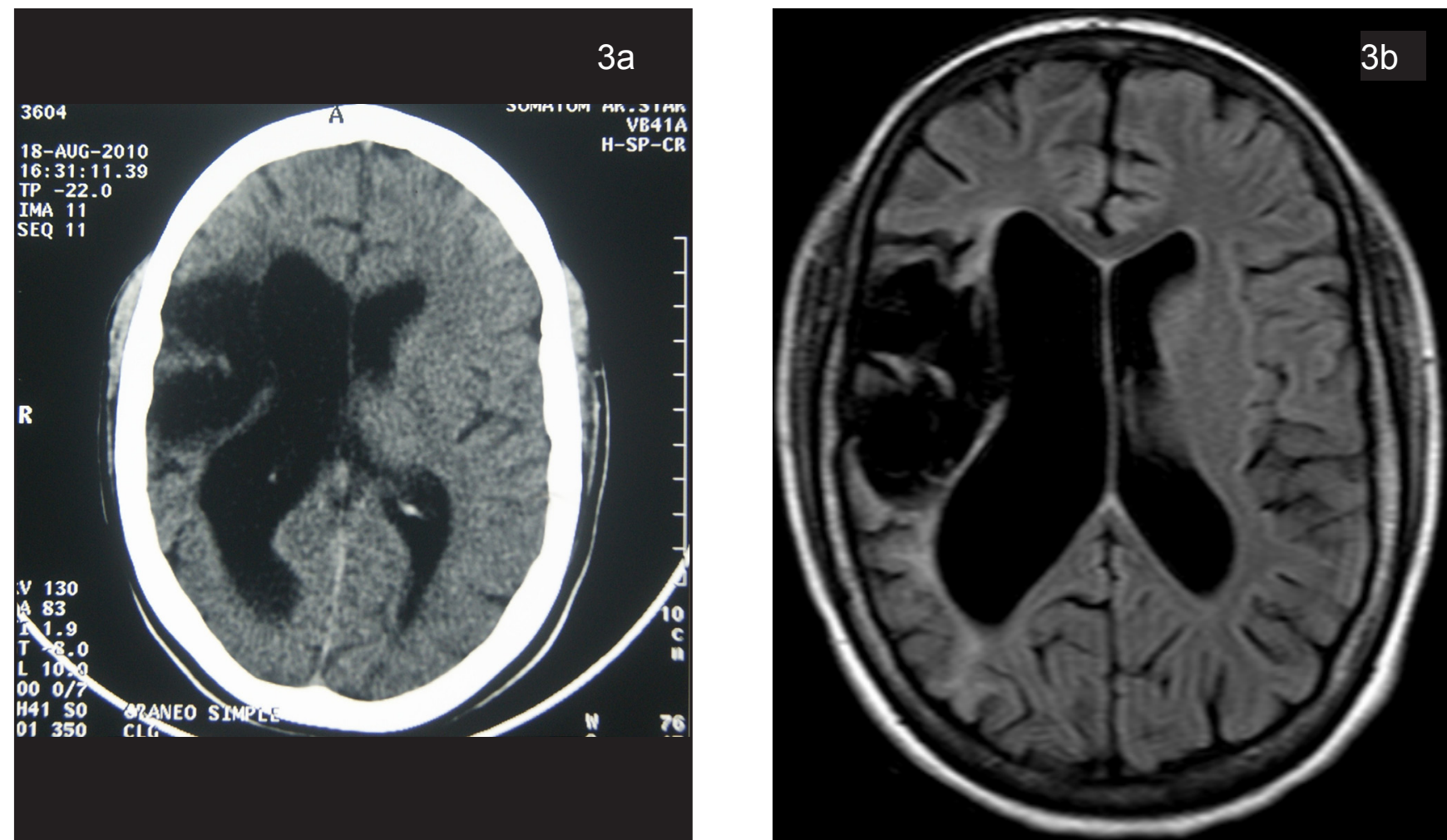

Figura 3. Imágenes diagnósticas del cerebro: (a) TAC de cráneo (18/10/2010): extensa encefalomalacia temporoparietal derecha con dilatación compensatoria del ventrículo lateral del mismo lado. (b) RM cerebro (16/4/2011): la misma lesión, sin evidencia de nuevas lesiones isquémicas.

de éstos sólo 35 correspondieron a Latinoamérica, con un claro predominio de Chile y sin ningún caso colombiano (3). Posteriormente, se han descrito en Colombia seis casos, cinco niños (cuatro con enfermedad moyamoya y uno asociado con síndrome de Down) y una mujer adulta con anemia de células falciformes (4-6). La concurrencia de enfermedad de Graves y síndrome moyamoya es rara. En una reciente revisión, Ohba et al. encontraron únicamente 31 casos en la literatura escrita en inglés y en sólo tres de éstos (9.7\%) los cambios fueron unilaterales (1). En China, Li et al. encontraron una prevalencia de $1.4 \%$ (21 pacientes) de la enfermedad de Graves en la población con enfermedad y síndrome moyamoya (1493 pacientes estudiados durante 10 años) (7).

El síndrome moyamoya es un trastorno vascular cerebral que predispone a los pacientes afectados al infarto debido a la estenosis progresiva de las arterias carótidas internas y sus ramas proximales. La disminución del flujo sanguíneo 
en las arterias principales de la circulación anterior del cerebro lleva al desarrollo compensatorio de una vasculatura colateral formada por pequeños vasos localizados cerca a la punta de la carótida, sobre la superficie cortical, leptomeninges y ramas de la arteria carótida externa que irrigan la duramadre y la base del cráneo. Rara vez este proceso involucra la circulación posterior, incluyendo a la arteria basilar y las arterias cerebrales posteriores (1).

Fue descrito por primera vez en 1957 por Takeuchi y Shimizu (2) como una "hipoplasia" bilateral de las arterias carótidas internas asociada a una red de vasos colaterales anormalmente dilatados. En 1969 se le añadió el nombre moyamoya, debido a la apariencia brumosa de estos vasos en la angiografía cerebral como la de una bocanada de humo de cigarrillo (lo que significa moyamoya en japonés) (2).

El síndrome moyamoya se asocia más frecuentemente con anemia de células falciformes, neurofibromatosis tipo I (enfermedad de von Recklinghausen), irradiación terapéutica craneal y síndrome de Down. Es rara su asociación con anormalidades cardiacas congénitas, estenosis de la arteria renal, hemangiomas cervicofaciales gigantes e hipertiroidismo (2).

En los 31 casos de enfermedad de Graves y estenosis u oclusión de las arterias intracraneales analizados por Ohba et al. (1) se encontró que esta asociación es más frecuente en mujeres (90.3\%) jóvenes (entre 10 y 54 años con un promedio de 29.3 años), al igual que el caso descrito por nosotros. En el $96.8 \%$ de los casos hubo tirotoxicidad cuando ocurrió el evento cerebral isquémico. En la serie de pacientes chinos también predominó el sexo femenino (76.2\%) y la mayoría presentaba hipertiroidismo $(85.7 \%)$ (7). El predominio del sexo femenino refleja la epidemiología de la enfermedad de Graves (8); de hecho cuando se analiza a toda la población con moyamoya, en la China la relación mujer a hombre fue de 1:1 $(9,10)$. En la mayoría de los casos los síntomas isquémicos mejoraron al tiempo con la normalización de los niveles de hormonas tiroideas $(1,7)$. El espectro clínico va desde el paciente asintomático (11) hasta la presentación rápidamente progresiva de infartos cerebrales masivos bilaterales fatales (12). Se ha descrito infarto cerebral durante la recaída del hipertiroidismo (1). En $90 \%$ de los casos se demuestran los infartos cerebrales en las imágenes diagnósticas del cerebro y la estenosis u oclusión bilateral de la porción terminal de la arteria carótida interna se comprobó en $90.3 \%$ de los estudios angiográficos. Los vasos en forma de red se vieron en $61.3 \%$ y cambios unilaterales sólo en $9.7 \%$ de los pacientes (1). Los cambios angiográficos pueden ser reversibles (13).

Los tratamientos utilizados en los pacientes afectados por estas dos patologías incluyen la terapia antitiroidea en el $86.2 \%$ y tratamiento quirúrgico en $35.5 \%$. Los pacientes tratados quirúrgicamente fueron, casi todos, tratados con terapia antitiroidea (1).

Los procedimientos quirúrgicos utilizados en la enfermedad y síndrome moyamoya buscan la revascularización del tejido cerebral mediante técnicas directas e indirectas; en las primeras, se realiza una anastomosis entre una rama de la arteria carótida externa (usualmente la arteria temporal superficial) y una arteria cortical. Las técnicas indirectas utilizan tejido vascularizado irrigado por la arteria carótida externa (por ejemplo: duramadre, músculo temporal o la propia arteria temporal superficial), el cual se coloca en contacto directo con el cerebro, lo que lleva a la formación de nuevos vasos en la corteza cerebral subyacente. La pialsinangiosis, cirugía en la cual se pone en contacto directo a la arteria temporal superficial con la corteza cerebral, es el método de revascularización indirecta preferido en la actualidad (2).

La mayoría de los pacientes con enfermedad de Graves y síndrome moyamoya se recuperan de sus síntomas neurológicos después del tratamiento médico o quirúrgico $(1,7)$, contrario a lo ocurrido en nuestra paciente que quedó con graves secuelas neurológicas. Debido al escaso número de casos descritos, no es posible establecer cuál es la mejor terapia para estos pacientes $(1,7)$.

No se ha determinado cuál es el mecanismo que lleva a la oclusión o estenosis de las arterias intracraneales en la enfermedad de Graves. Sin embargo, existen varias hipótesis (1). En primer lugar las hormonas tiroideas pueden aumentar la sensibilidad vascular al sistema nervioso simpático e inducir cambios patológicos en las paredes arteriales. Otra hipótesis (14) señala que un mecanismo inmunológico puede estar involucrado en la patogénesis de la enfermedad, debido a que se han descrito cambios de vasculitis cerebral; esta hipótesis tiene a su favor el hecho que la enfermedad de Graves es de origen autoinmune y que otras enfermedades de este tipo como el lupus eritematoso sistémico, el síndrome antifosfolípidos y la colitis ulcerativa se han informado asociadas al síndrome moyamoya. Una tercera teoría (15) sindica a la ateroesclerosis como el vínculo clave entre la enfermedad de Graves y el síndrome moyamoya debido a hiperhomocisteinemia inducida por la tirotoxicosis. Los agentes antitiroideos, como el propiltiouracilo, pueden inducir vasculitis asociadas a cambios en las arterias intracraneales. Finalmente, factores genéticos también pueden contribuir en la patogénesis de esta asociación (7).

En conclusión, el infarto cerebral sería el resultado de los cambios hemodinámicos cerebrovasculares inducidos por la tirotoxicosis. En el estado tirotóxico hay una excesiva producción de hormonas tiroideas que aumentan el metabolismo cerebral y el consumo de oxígeno, lo que produce una alteración en la perfusión cerebral. Además, existe un estado de hipercoagulabilidad que favorece los cambios isquémicos. Esto explicaría el porqué son más frecuentes los eventos isquémicos que los hemorrágicos.

A pesar de lo raro del caso presentado, en todo paciente con tirotoxicosis secundaria a la enfermedad de Graves que presente síntomas neurológicos focales agudos, debería investigarse la posibilidad de síndrome moyamoya. 


\section{Contribución de los autores}

Jairo Lizarazo, Freddy Niño, Hugo Alvarado y Noé Castro fueron los directos responsables del manejo de la paciente.

Jairo Lizarazo es el responsable de la redacción del artículo.

Freddy Niño, Hugo Alvarado y Noé Castro se encargaron de la revisión crítica del manuscrito.

\section{Agradecimientos}

A Shigeo Ohba por facilitarnos su artículo. A Elizabeth Castañeda por su revisión crítica del manuscrito.

\section{Conflicto de intereses}

Los autores declaramos que no tenemos conflicto de intereses.

\section{Financiación}

Ninguno de los autores recibió financiación alguna.

\section{Referencias}

1. Ohba S, Nakagawa T, Murakami H. Concurrent Graves' disease and intracranial arterialstenosis/occlusion: special considerations regarding the state of thyroid function, etiology, and treatment. Neurosurg Rev 2011; 34: 297-304.

2. Scott RM, Smith ER. moyamoya disease and moyamoya syndrome. $N$ Engl J Med 2009; 360: 1226-37.

3. Goto Y, Yonekawa Y. Worldwide distribution of moyamoya disease. Neurol Med Chir (Tokyo) 1992; 32: 883-6.

4. Borrero L, Henao L, Hernández J. Síndrome de moyamoya: diagnóstico mediante resonancia magnética. Rev Colomb Radiol 1997; 8: 115-8.
5. Espinosa E, Ortiz A,Ardila S, Cabarcas L, Mancilla N. Síndrome y enfermedad de moyamoya. Acta Neurol Colomb 2011; 27: 165-71.

6. Granados A, Hernández OH O, Guerra A, Uribe CS. Síndrome de moyamoya y complicaciones neurológicas en un paciente con enfermedad de células falciforme. Acta Neurol Colomb 2012; 28: 49-54

7. Li D, Yang W, Xian P, Liu P, Bao X, Zong R, Duan L. Coexistence of moyamoya and Graves' diseases: The clinical characteristics and treatment effects of 21 Chinese patients. Clin Neurol Neurosurg 2013; S0303-8467(13): 00071-1. 10.1016/j.clineuro.2013.02.018.

8. Brent GA. Graves' disease. New Eng J Med 2008; 358: 2594-2605.

9. Bao XY, Duan L, Li DS, Yang WZ, Sun WJ, Zhang ZS, Zong R, Han C. Clinical features, surgical treatment and long-term outcome in adult patients with moyamoya disease in China. Cerebrovasc Dis 2012; 34: 305-13.

10. Duan L, Bao XY, Yang WZ, Shi WC, Li DS, Zhang ZS, Zong R, Han C, Zhao F, Feng J. moyamoya disease in China: its clinical features and outcomes. Stroke 2012; 43: 56-60.

11. He Y, Zhou Q, He M. An asymptomatic moyamoya disease. Autopsy case and literature review. Am J Forensic Med Pathol 2010; 31: 77-9.

12. Hsu SW, Chaloupka JC, Fattal D. Rapidly progressive fatal bihemispheric infarction secondary to moyamoya syndrome in association with Graves thyrotoxicosis. Am J Neuroradiol 2006; 27: 643-7.

13. Utku U, Asil T, Celik Y, Tucer D. Reversible MR angiographic findings in patient with autoimmune Graves disease. Am J Neuroradiol 2004; 25: 1541-3.

14. Panegyres PK, Morris JG, O’Neill PJ, Balleine R. moyamoya-like disease with inflammation. Eur Neurol 1993; 33: 260-263.

15. Sasaki T, Nogawa S,Amano T. Co-morbidity of moyamoya disease with Graves' disease. Report of three cases and a review of the literature. Intern Med 2206; 45: 649-653. 